\title{
La Educación superior durante la contingencia sanitaria COVID-19: Uso de las TIC como herramientas de aprendizaje. Caso de estudio: alumnos de la Facultad de Contaduría y Administración
}

Higher Education during the health contingency COVID-19: Use of ICTs as learning tools. Case study: students of the Faculty of Accounting and Administration

\author{
Alma Lilia Sapién Aguilar. Universidad Autónoma de Chihuahua. México. \\ lsapien@uach.mx \\ $[\mathrm{CV}]$ \\ Laura Cristina Piñón Howlet. Universidad Autónoma de Chihuahua. México. \\ lpinon@uach.mx \\ [CV] 10
}

María del Carmen Gutiérrez Diez. Universidad Autónoma de Chihuahua. México.

cgutierr@uach.mx

$[\mathrm{CV}]$

José Luis Bordas Beltrán. Universidad Autónoma de Chihuahua. México.

jbordas@uach.mx

$[\mathrm{CV}]$ (1)

Cómo citar este artículo / Referencia normalizada

Sapién Aguilar, A. L., Piñón Howlet, L. C., Gutiérrez-Diez, M. C. y Bordas Beltrán, J. L. (2020).

La Educación superior durante la contingencia sanitaria COVID-19: Uso de las TIC como herramientas de aprendizaje. Caso de estudio: alumnos de la Facultad de Contaduría y Administración. Revista Latina de Comunicación Social, 78, 309-328. https://www.doi.org/10.4185/RLCS-2020-1479

\section{RESUMEN}

Las Tecnologías de la información y la comunicación en el sector educativo tienen una relevancia importante en el proceso de enseñanza aprendizaje de los estudiantes y en el conocimiento de las competencias para la vida. El objetivo de la investigación fue analizar el uso de las TIC como herramientas de aprendizaje ante la contingencia COVID-19 en los alumnos de la Facultad de Contaduría y Administración de la Universidad Autónoma de Chihuahua en México. La naturaleza de esta investigación fue cuantitativa y el tipo de investigación fue de forma aplicada, el alcance fue descriptivo y la investigación de campo, el diseño fue no experimental transeccional descriptivo, la 
técnica de recolección de información fue mediante un cuestionario. Los resultados revelaron una correlación positiva hacia las tecnologías de información y comunicación, que se evidencia en diferentes aspectos examinados: estilo de aprendizaje, dominio en el uso de las TIC, redes sociales, programa educativo, nivel de satisfacción de contar con los recursos tecnológicos apropiados, entre otras; en todas ellas se manifiesta una predisposición favorable. Las ventajas que los estudiantes consideran con el uso de las TIC son la disponibilidad de información y aprendizaje colaborativo. De las desventajas que se identificaron fueron las fallas en la conexión de Internet, tienen acceso a muchas distracciones durante la clase y consideran que tienen un menor aprendizaje. Uno de los hallazgos más importantes de esta investigación fue que los estudiantes tienen muy buen dominio de las TIC para manejar las herramientas tecnológicas utilizadas durante la contingencia sanitaria del COVID-19.

PALABRAS CLAVE: TIC; COVID-19; uso; aprendizaje; educación a distancia; estudiantes.

\begin{abstract}
Information and communication technologies in the educational sector have an important relevance in the teaching-learning process of students and in the knowledge of life skills. The objective of the research was to analyze the use of ICT as learning tools in the face of the COVID-19 contingency in the students of the Faculty of Accounting and Administration of the Autonomous University of Chihuahua in Mexico. He nature of this research was quantitative and the type of research was applied, the scope was descriptive and the field research, the design was non-experimental descriptive transectional, the technique of gathering information was through a questionnaire. The main results showed a positive correlation towards information and communication technologies, which is shown in various aspects studied: learning style, mastery of the use of ICT, social networks, educational program, level of satisfaction with having technological resources appropriate; in all of them a favorable predisposition is manifested. The advantages that students consider with the use of ICT are the availability of information and collaborative learning. Of the disadvantages that were identified were the failures in the Internet connection, they have access to many distractions during class and consider that they have less learning. One of the most important findings of this research was that students have very good mastery of ICT to manage the technological tools used during the COVID-19 health contingency.
\end{abstract}

KEYWORDS: ICT; COVID-19; use; learning; distance education; students.

\title{
CONTENIDO
}

1. Introducción. 2. Objetivos. 3. Metodología. 4. Discusión/Resultados. 5. Conclusiones. 6. Recomendaciones 7. Bibliografía

\section{Introducción}

Las tecnologías de la información y de la comunicación (TIC) son un recurso fundamental de desarrollos sin precedentes en el mundo actual generando grandiosas innovaciones en la humanidad, en la ciencia y en el gobierno. Las sociedades son lo que son por el aumento de la importancia de la tecnología, sin embargo, no se debe olvidar que las TIC no resolverán todos los problemas y que, en el ámbito educativo, son instrumentos que permiten emplear diferentes caminos y acciones por probar, donde el principal reto es el desarrollo de habilidades y destrezas que son aptos de computarizarse como la creatividad o la inteligencia social. (Cobo, 2016) 
En la actualidad se está ante el supuesto de la inclusión de las TIC en el ámbito educativo: si se carece de conocimientos técnicos en el uso y aplicación de los instrumentos digitales, será complejo incorporarlas en el ámbito educativo. La deficiencia que tienen los docentes en el uso de las herramientas tecnológicas, le dificultará emplearlas con efectividad en el proceso educativo con sus estudiantes. (Fernández-Cruz y Fernández-Díaz, 2016)

En México, en el mes de marzo del 2020, fue publicado por el Consejo de Salubridad General la epidemia por enfermedad SARS-CoV2 (COVID-19) como emergencia sanitaria por causas de fuerza mayor (Consejo de Salubridad General, 2020).

Ante la factible transmisión del virus y la amenazadora exposición de la población a este, ya sea por el contacto con personas infectadas y/o por estar expuestos a superficies u objetos contagiosos; fue que la Secretaría de Educación Pública (SEP), al recibir las indicaciones e información por parte de la Secretaría de Salud, tomó el acuerdo de suspender las clases en las instituciones dependientes de la SEP para proteger a la comunidad estudiantil; tal acuerdo fue difundido en el Diario Oficial de la Federación (DOF) (Secretaría de Educación Pública, 2020); actualmente, la suspensión continúa y así seguirá hasta que de acuerdo al semáforo epidemiológico pase de rojo hasta llegar al color verde en dónde se permitirá el desarrollo de todas las actividades, incluidas las escolares (Gobierno de México, 2020).

Las Instituciones de Educación Superior en su modalidad presencial se enfrentan ante el hecho de continuar con el desarrollo de los programas educativos en las instituciones superando barreras como lo son la falta de equipo, falta de capacitación, resistencia a adoptar nuevas tecnologías o métodos entre otras, situación derivada de las crisis por la pandemia del COVID-19, los estudiantes deben continuar con su aprendizaje desde sus hogares a través del uso de las Tecnologías de Información y Comunicaciones (TIC). Los alumnos y profesores se encuentran ante la problemática de adaptarse a estos cambios y tener que aprender nuevas formas de transmitir el conocimiento. Esta etapa de transición tendrá un cambio en las instituciones educativas debido a que el proceso de enseñanzaaprendizaje será diferente al tradicional con el uso de las TIC las cuales han adquirido gran relevancia en el entorno educativo con grandes retos en tiempos sin precedentes, en donde la educación está ante una transformación completa.

En atención a la Declaratoria de Emergencia Sanitaria publicada en el Diario Oficial de la Federación por la contingencia del COVID-19, la Universidad Autónoma de Chihuahua (UACH) puso a disposición de toda la comunidad académica la Plataforma Educativa Institucional Moodle, con el fin de dar continuidad a las actividades académicas de todos los programas educativos del Nivel Técnico, Licenciatura y Posgrado, así mismo, los servicios institucionales en línea como son las Videoconferencias Webex y la Suite de Google como apoyo a las actividades académicas.

La suspensión provocó gran incertidumbre en las instituciones de educación en todos los niveles. En este tiempo de confinamiento dentro de la comunidad universitaria, se identificaron diferentes problemas que los estudiantes y profesores debieron sortear como lo son: poca comunicación entre profesores y alumnos, la falta de equipo de computo, de capacitación y de conexiones a internet además de tener que manejar nuevas tecnologías de enseñanza-aprendizaje para continuar con el periodo escolar a distancia.

Para dar continuidad con el semestre bajo la educación virtual, fue necesario, el uso de equipo de cómputo; el que tenían acceso en la escuela ya no estaba disponible, los estudiantes debieron buscar alternativas para atender las clases ya que algunos no contaban con uno, o bien las herramientas tecnológicas con las que contaban eran obsoletas; otros hicieron uso de su teléfono inteligente (con 
ciertas limitaciones). Algunos alumnos, a pesar de tener una computadora, la comparten con los demás miembros de la familia y esto les limita su uso. La falta de internet fue otra dificultad para ellos, algunos no contaban con el servicio y se vieron en la necesidad de buscar diferentes alternativas o de contratarlo, sin embargo, hoy en día, no todos tienen la capacidad económica para invertir en este servicio; aunado a que no en todas las localidades de residencia de estos llega la señal de internet. Otro problema fue la falta de conocimientos y habilidades para el manejo de herramientas digitales básicamente los alumnos utilizan el internet, pero un número limitado recurre a bases de datos electrónicas para sus investigaciones, las herramientas que utilizan son las de la suite de Office y solo unos cuantos habían tenido acceso a plataformas como Moodle, Meet o Zoom entre otras.

Por lo anterior; se plantearon las siguientes preguntas de investigación: 1) ¿cuál es el uso que tienen las TIC como herramientas de aprendizaje ante la contingencia COVID-19 en los alumnos de la Facultad de Contaduría y Administración (FCA) de la Universidad Autónoma de Chihuahua?; 2) ¿cuál es la relación del uso de las TIC con el género, dominio de las TIC, estilo de aprendizaje, características de las TIC y nivel de satisfacción?; 3)¿cuál es el grado de correlación del uso de las TIC con los programas educativos con relación al dominio, redes sociales, semestre, herramientas usadas, continuación de clases virtuales y lugar de conexión?; 4) ¿cuáles son las ventajas y desventajas de las TIC como herramientas de aprendizaje en los alumnos?

El objetivo general de la investigación fue analizar el uso de las TIC como herramientas de aprendizaje ante la contingencia COVID-19 en los alumnos de la Facultad de Contaduría y Administración (FCA) de la Universidad Autónoma de Chihuahua. Los objetivos específicos fueron: 1) Identificar la relación del uso de las TIC con género, dominio de las TIC, estilo de aprendizaje, características de las TIC y nivel de satisfacción; 2) distinguir el grado de correlación de los programas educativos con dominio de las TIC, redes sociales, semestre, herramientas usadas, continuación de clases virtuales y lugar de conexión y 3) determinar las ventajas y desventajas de la TIC como herramientas de aprendizaje en los alumnos.

\section{Marco teórico:}

Las TIC son un conjunto de tecnologías que permiten la adquisición, elaboración, almacenamiento, tratamiento, comunicación, registro y presentación de información, en forma de voz, imágenes y datos contenidos en señales de naturaleza acústica, óptica o electromagnética. Las TIC incluyen la electrónica como tecnología base que soporta el desarrollo de las telecomunicaciones, la informática y el audiovisual, pero también incorporan toda una serie de elementos innovadores al servicio de la educación y el aprendizaje significativo. (Cacheiro, 2014)

Las TIC desde el punto de vista educativo, como lo menciona Mendoza (2018), es una de las innovaciones más significativas en el campo de la educación, porque permiten apoderarse de un espacio efectivo en el contexto educativo en el ámbito internacional. El uso de la tecnología en la educación ha sido clave para el desarrollo y la creación de tecnologías educativas en el ejercicio diario en las aulas.

A juicio de Astudillo et. ál. (2018), el papel primordial de las TIC es reforzar y transformar las prácticas educativas. Para ello es necesario apropiarse de las redes de aprendizaje y vislumbrar que todos los involucrados deben considerarse como semejantes. Porque la idea esencial es contribuir, discutir, debatir y abrir un proceso de comunicación efectiva con la colaboración y la creación de conocimientos. De esta manera los procesos de aprendizaje resultan motivadores y significativos si 
se consigue realizar un cambio acorde a las necesidades actuales. Este trabajo debe darse entre profesores y estudiantes, para que se logren los objetivos planteados.

Silva, García, Guzmán y Chaparro (2016) mencionan que hoy en día la combinación de las Tecnologías de la Información y Comunicación (TIC) es un recurso habitual en los ambientes de innovación educativa que, cada vez más, propone al aumento de las acciones basadas en la interacción y la creación compuesta de conocimientos (Avello-Martínez y Duart, 2016). En este entorno se ha demostrado que los Learning Management System (LMS) incrementan la participación y la cooperación, entre los actores del proceso de enseñanza-aprendizaje (Perkins y Pfaffman, 2006; Vázquez y Burrial, 2017; Yanacón-Atía y Menini, 2018). Sin embargo, la interacción y colaboración se han convertido en una variable importante (Smith y Xu, 2016) dentro del contexto formativo creado a partir del Espacio Europeo de Educación Superior (EEES), lo que ha implicado la implementación intensiva de plataformas de enseñanza-aprendizaje en línea como Moodle (Sistema de Gestión de Aprendizaje), para propiciar la creación de Entornos Virtuales de Aprendizaje (EVA), el aprendizaje colaborativo y el aprendizaje mixto o blended-learning. (Vuopala, Hyvönen y Järvelä, 2016; Coyago, Puente y Jiménez, 2017)

En una sociedad actual, donde la educación se apoya en las tecnologías, el acceder al conocimiento está fundamentada en dos elementos principales: a) los soportes tecnológicos y b) la comunicación, que se han transformado en la herramienta básica de la gestión de conocimiento (Moreno, 2016). Por otro lado, a través del uso de las computadoras, la sociedad globalizada se relaciona, por lo que el compartir información y conocimiento son por medio de aparatos físicos y de las telecomunicaciones, esto quiere decir, que inclusive la educación, utiliza los equipos de cómputo para guiarnos a la virtualización en todas las áreas de la sociedad.

Como ya se ha señalado, el desarrollo de las TIC ha originado toda una revolución en el área de las comunicaciones y en este momento brinda nuevas formas de enseñanza. Internet fue, de inicio, una herramienta que se utilizaba para comunicarse entre computadoras y departamentos, solo disponibles para las personas familiarizadas con las líneas de comandos. Gracias al desarrollo del hipervínculo en la Web lo transformó en una herramienta amigable que permite que un documento incluya elementos que proviene de otros documentos y que a su vez nos lleve a otros. (St-pierre y Kustcher, 2001)

La implementación de las TIC ha considerado que para las instituciones educativas estas dejen de ser una opción, y los impulsos de los países e instituciones se enfoquen en la generación e implementación de propuestas que involucren la máxima utilización de las tecnologías en los procesos docentes. (Severin, 2010)

Cacheiro (2018) señala que para que sea indiscutible el uso de las TIC en el proceso enseñanza aprendizaje, es necesario una manera de pensar diferente en el papel de los profesores, pasando de ser transmisor a guía. Por lo tanto, esto propicia una evolución en los estudiantes, donde toma una participación activa en esta transformación.

El problema de adopción de las TIC en los procesos educativos parece ser estructural. Existe interés por parte de los profesores, pero en las instituciones de educación superior falta una planeación estratégica para la adopción de las herramientas tecnológicas, aunque en las instituciones tengan la infraestructura propicia; sin embargo, falta la capacitación a los profesores lo cual conlleva a la exigencia de una formación pedagógica institucionalizada y sistemática, cuyo objetivo sea el de promover nuevas competencias entre los docentes. (Vera et ál., 2014) 
La educación virtual apoya a un mejor aprendizaje porque en diferentes áreas se considera que la educación externa, a la tradicional es más propicia tomando en cuenta la revolución del conocimiento, esto ha generado que los alumnos desarrollen de forma autónoma aprendizaje, además de ser considerados nativos digitales por tener acceso a la información en los diferentes dispositivos a su alcance, esto conlleva a pensar en una estrategia metacognitiva en el área de las TIC. En primer lugar, han transformado el proceso cognitivo y, como lo señala, se ha generado un nuevo tipo de inteligencia, la inteligencia distribuida que se define como "la inteligencia no es una propiedad atribuida a las mentes de los individuos, sino que se distribuye entre las personas, y entre las personas y las herramientas físicas y los sistemas simbólicos" (Herrero y Brown, 2010), esto ha llevado a nuevos paradigmas y nuevas formas de enseñanza y aprendizaje, como el conectivismo, que intenta demostrarlo a través de las redes de aprendizaje, las redes sociales, el autoaprendizaje y entornos personales de aprendizaje, además del uso de las TIC, los estudiantes pueden generar su conocimiento de forma autónoma, trascendiendo su construcción del aula virtual y ser evaluados por ellos y sus compañeros, simplemente dejando al maestro como mediador de lo aprendido. (Gallego Torres, 2017)

En segundo lugar, debe considerarse que, debido al uso de entornos computacionales, según Karl Stephen (Sierra, Carrascal y Buelvas, 2014) se puede dividir en dos tipos: Sistema de contenedores, que es cómo se llaman frecuentemente los entornos de aprendizaje virtual, son repositorios de información preestablecidos y centrados en el entorno, y los recursos ofrecidos por las diferentes plataformas, también del sistema de contenido donde los usuarios proveen y comparten información y contenido, alcanzando una apropiación del curso por parte de los estudiantes que participan en el proceso y no solo como destinatarios de conocimiento, sino no, como pro-cosumidores, eso significa que consumen y producen la información necesaria para lograr el objetivo de aprendizaje requerido por el curso, esto se logra con la articulación del contenido planteado por los estudiantes y con las actividades, evaluaciones y comentarios de los profesores, que utilizan herramientas asincrónicas como foros, blogs, etc.

\section{Metodología}

El trabajo de investigación se desarrolló en la Facultad de Contaduría y Administración (FCA) de la Universidad Autónoma de Chihuahua (UACH) de abril a junio de 2020. El enfoque de esta investigación fue de naturaleza cuantitativa de tipo aplicada. El diseño fue de tipo no experimental pues no se manipularon las variables. Transeccional porque se recolectaron los datos en un tiempo único y descriptiva ya que se pretendió conocer la situación actual y estuvo orientada a medir las variables.

El instrumento de recolección de información fue un cuestionario en Google Forms (anexo cuestionario), especialmente diseñado para evaluar la variable Uso de las TIC. El cuestionario fue integrado por un total de 30 preguntas cerradas; en las cuales, como respuesta se solicitaba un grado de acuerdo o desacuerdo, según la escala de Likert.

En la Tabla 1 se muestra, a nivel de detalle, cómo se realizó la sistematización de la variable Uso de las TIC. Como se puede observar en la Tabla 1 se consideraron tres dimensiones; datos generales, manejo de las TIC y herramientas. El cuestionario fue validado a través de una prueba piloto y la confiabilidad del instrumento para cada una de sus dimensiones fue calculada mediante el coeficiente de consistencia interna de Cronbach, que reportó resultados aceptables $(\alpha \geq 0,70)$. 
Tabla 1. Sistematización de la variable de estudio

\begin{tabular}{|c|c|c|c|}
\hline Variable & Dimensiones & Indicadores & Ítems \\
\hline \multirow{3}{*}{ Uso de las TIC } & Datos generales & $\begin{array}{ll}- & \text { Programa educativo } \\
- & \text { Semestre en curso } \\
- & \text { Continuidad en línea } \\
- & \text { Lugar de acceso de Internet } \\
- & \text { Género } \\
- & \text { Edad } \\
- & \text { Estado Civil }\end{array}$ & $1-2-3-4-5-6$ \\
\hline & Manejo de las TIC & $\begin{array}{ll}- & \text { Dominio de las TIC por parte del } \\
& \text { alumno } \\
- & \text { Estilo de aprendizaje } \\
- & \text { Dominio de las TIC por parte del } \\
& \text { maestro } \\
-\quad & \text { Conexión a Internet } \\
- & \text { Frecuencia de uso de las TIC }\end{array}$ & $\begin{array}{c}7-8-9-10-11-14-15 \\
-17-18-19-20-21-22 \\
-23-24-25\end{array}$ \\
\hline & Herramientas & $\begin{array}{ll}- & \text { Características } \\
- & \text { Nivel de satisfacción } \\
- & \text { Redes sociales } \\
- & \text { Ventajas } \\
- & \text { Desventajas }\end{array}$ & $\begin{array}{c}12-13-16-26-2728- \\
29-30\end{array}$ \\
\hline
\end{tabular}

El tipo de muestreo fue no probabilístico, se tomaron en cuenta a todos los alumnos inscritos en el semestre enero-junio de 2020 en la modalidad presencial. La población de interés que se tomó fue de 4,522 alumnos de todos los programas educativos que ofrece la Facultad de Contaduría y Administración: Licenciado en Administración de Empresas (LAE), Contador Público (CP), Licenciado en Administración Financiera (LAF), Licenciado en Administración Gubernamental (LAG) y Licenciado en Administración de Tecnologías de la Información y Comunicación (LATIC); la muestra se calculó con un nivel de confianza del 95\%. La aplicación de dicha ecuación dio como resultado la obtención de una muestra total de 1,198 alumnos, se dividió a la población en grupos según la Licenciatura en la que estaban inscritos por compartir características afines, se seleccionaron a los alumnos de manera voluntaria. La información básica fue proporcionada por la coordinación académica de la modalidad presencial de la FCA, de donde se obtuvo la muestra.

Después obtener los resultados derivados del cuestionario, se procesó la información en Excel y SPSS V.22, procediendo a realizar tablas de análisis de la información obtenida, tablas de frecuencias y correlaciones entre las preguntas que proyectaron descubrimientos más representativos en cuanto al uso de las Tecnologías de información y Comunicación después de haber pasado de la educación tradicional a la educación a distancia debido a la contingencia sanitaria. Por último, se elaboró un cruce entre la información obtenida de manera cuantitativa. En esta investigación se muestran los resultados de las preguntas con mayor relevancia sobre el tema abordado.

\section{Resultados}

Los resultados se presentan de acuerdo con la metodología planteada, así como el análisis de los datos recopilados a través del cuestionario sobre el uso de las Tecnologías de Información y Comunicaciones (TIC) ante la pandemia del COVID-19 en la FCA de la UACH en los diferentes programas educativos que ofrece, además de datos generales como género, edad y discapacidad. Los cuáles se presentan divididos de la siguiente manera: 
RLCS, Revista Latina de Comunicación Social, 78, 309-328

[Investigación] DOI: 10.4185/RLCS-2020-1479| ISSN 1138-5820| Año 2020

Tabla 2. Perfil de las muestras

\begin{tabular}{|c|c|c|c|}
\hline $\begin{array}{l}\text { Programa } \\
\text { Educativo }\end{array}$ & Género & Edad & Discapacidad \\
\hline \multirow{4}{*}{ C.P. } & \multirow{2}{*}{ 63.6\% Mujeres } & $40.7 \%>17<19$ años & $0 \%$ Auditiva \\
\hline & & $43.8 \%$ entre 20 y 22 años & $0.3 \%$ Motriz \\
\hline & \multirow{2}{*}{$36.4 \%$ Hombres } & $9.9 \%$ entre 23 y 25 años & $10.5 \%$ Visual \\
\hline & & $5.6 \%$ más de 25 años & $89.2 \%$ Ninguna \\
\hline \multirow{4}{*}{ L.A.E. } & \multirow{2}{*}{$67.8 \%$ Mujeres } & $34.9 \%$ entre 17 y 19 años & $0.8 \%$ Auditiva \\
\hline & & $50.4 \%$ entre 20 y 22 años & $1.1 \%$ Motriz \\
\hline & \multirow{2}{*}{$32.2 \%$ Hombres } & $12.8 \%$ entre 23 y 25 años & $8.2 \%$ Visual \\
\hline & & $1.9 \%$ más de 25 años & $89.9 \%$ Ninguna \\
\hline \multirow{4}{*}{ L.A.F. } & \multirow{2}{*}{$56.4 \%$ Mujeres } & $37.5 \%$ entre 17 y 19 años & $0.7 \%$ Auditiva \\
\hline & & $49.1 \%$ entre 20 y 22 años & $0.3 \%$ Motriz \\
\hline & \multirow{2}{*}{$43.6 \%$ Hombres } & $12 \%$ entre 23 y 25 años & $7.2 \%$ Visual \\
\hline & & $1.4 \%$ más de 25 años & $91.8 \%$ Ninguna \\
\hline \multirow{4}{*}{ L.A.G. } & \multirow{2}{*}{$60.2 \%$ Mujeres } & $43.9 \%$ entre 17 y 19 años & $1.6 \%$ Auditiva \\
\hline & & $38.2 \%$ entre 20 y 22 años & $0.8 \%$ Motriz \\
\hline & \multirow{2}{*}{$39.8 \%$ Hombres } & $14.6 \%$ entre 23 y 25 años & $8.9 \%$ Visual \\
\hline & & $3.3 \%$ más de 25 años & $88.6 \%$ Ninguna \\
\hline \multirow{4}{*}{ L.A.T.I.C. } & \multirow{2}{*}{$33.3 \%$ Mujeres } & $33.3 \%$ entre 17 y 19 años & $2.2 \%$ Auditiva \\
\hline & & $43 \%$ entre 20 y 22 años & $1.1 \%$ Motriz \\
\hline & \multirow{2}{*}{$66.7 \%$ Hombres } & $18.3 \%$ entre 23 y 25 años & $11.8 \%$ Visual \\
\hline & & $5.4 \%$ más de 25 años & $84.9 \%$ Ninguna \\
\hline \multirow{4}{*}{ TOTAL } & \multirow{2}{*}{$60.4 \%$ Mujeres } & $37.9 \%$ entre 17 y 19 años & $0.8 \%$ Auditiva \\
\hline & & $46.5 \%$ entre 20 y 22 años & $0.7 \%$ Motriz \\
\hline & \multirow[t]{2}{*}{$39.6 \%$ Hombres } & $12.4 \%$ entre 23 y 25 años & $8.9 \%$ Visual \\
\hline & & $3.2 \%$ más de 25 años & $89.6 \%$ Ninguna \\
\hline
\end{tabular}

Fuente: Elaboración propia (2020)

La tabla 2 permite conocer por programa educativo el porcentaje de género, el rango de edades, así como existencia de algún tipo de discapacidad en los alumnos encuestados. Donde se puede observar que solamente en el programa educativo LATIC existe un mayor porcentaje de hombres que de mujeres, de igual forma únicamente el $3.2 \%$ son mayores de 25 años y el $89.6 \%$ no tiene alguna discapacidad. La mayor parte de la población de la Facultad son mujeres.

Tabla 3. Herramientas que se utilizan para consulta de información

\begin{tabular}{|l|c|c|c|c|c|}
\hline $\begin{array}{l}\text { Programa } \\
\text { educativo }\end{array}$ & Buscadores & Bases de datos & Redes Sociales & Blogs & Ninguna \\
\hline C.P. & 303 & 109 & 103 & 46 & 0 \\
\hline L.A.E. & 344 & 122 & 129 & 72 & 4 \\
\hline L.A.T.I.C. & 84 & 43 & 52 & 19 & 0 \\
\hline L.A.F. & 279 & 98 & 105 & 64 & 0 \\
\hline L.A.G. & 117 & 55 & 54 & 20 & 0 \\
\hline
\end{tabular}

Fuente: Elaboración propia (2020)

La tabla 3 muestra que el $94 \%$ de los encuestados utilizan buscadores y son los programas educativos LAE, CP y LAG los que hacen el mayor uso de ellas con una cantidad de 344, 303 y 279 alumnos por carrera respectivamente. En cuanto a las bases de datos y redes sociales usadas, representan entre el 36 y $37 \%$ respectivamente. Por último, los blogs representan solamente el 18\% como herramienta utilizada para consultar información. 
RLCS, Revista Latina de Comunicación Social, 78, 309-328

[Investigación] DOI: 10.4185/RLCS-2020-1479| ISSN 1138-5820| Año 2020

Tabla 4. Herramientas que están utilizando ante la contingencia COVID-19

\begin{tabular}{|l|c|c|c|c|c|}
\hline Herramientas & C.P. & L.A.E. & L.A.T.I.C. & L.A.F. & L.A.G. \\
\hline Plataforma Moodle & 185 & 239 & 76 & 176 & 93 \\
\hline Google Classroom & 137 & 146 & 25 & 130 & 39 \\
\hline Video conferencias por medio de la plataforma Meet & 278 & 311 & 74 & 251 & 109 \\
\hline Video conferencias por medio de la plataforma Zoom & 118 & 120 & 49 & 98 & 39 \\
\hline Correo Electrónico & 101 & 131 & 22 & 84 & 39 \\
\hline Nube computacional (Google Drive) & 64 & 69 & 29 & 53 & 25 \\
\hline Otro & 23 & 9 & 5 & 16 & 1 \\
\hline
\end{tabular}

Fuente: Elaboración propia (2020)

En la tabla 4 se puede observar las diferentes herramientas que los alumnos encuestados utilizan para sus clases a distancia por la contingencia COVID-19, en el programa educativo CP el $76 \%$ utilizan una o varias de estas herramientas, mientras que LAE cuenta con un $86 \%$ de uso en dichas herramientas. Los programas correspondientes a LATIC, LAF y LAG hacen uso de diferentes herramientas en un 23, 67 y $29 \%$ respectivamente. Siendo las herramientas más utilizadas las videoconferencias por medio de la plataforma Meet y el uso de la plataforma Moodle.

Tabla 5. Dominio de TIC por parte de los alumnos por Carrera y Género

\begin{tabular}{|c|c|c|c|c|c|c|}
\hline $\begin{array}{l}\text { Programa } \\
\text { educativo }\end{array}$ & Género & Malo & Regular & Bueno & Muy Bueno & Excelente \\
\hline \multirow{2}{*}{ C.P. } & Hombre & 9 & 9 & 34 & 40 & 26 \\
\hline & Mujer & 6 & 18 & 82 & 107 & 37 \\
\hline \multirow{2}{*}{ L.A.E. } & Hombre & 4 & 5 & 35 & 48 & 26 \\
\hline & Mujer & 7 & 13 & 74 & 77 & 35 \\
\hline \multirow{2}{*}{ L.A.T.I.C. } & Hombre & 9 & 1 & 12 & 14 & 27 \\
\hline & Mujer & 0 & 1 & 5 & 11 & 14 \\
\hline \multirow{2}{*}{ L.A.F. } & Hombre & 7 & 9 & 37 & 51 & 23 \\
\hline & Mujer & 5 & 8 & 55 & 67 & 29 \\
\hline \multirow{2}{*}{ L.A.G. } & Hombre & 1 & 2 & 18 & 21 & 7 \\
\hline & Mujer & 2 & 4 & 32 & 22 & 14 \\
\hline
\end{tabular}

Fuente: Elaboración propia (2020)

La tabla 5 muestra el desempeño de los alumnos en cuanto al dominio que poseen de las TIC, quedando el programa educativo $\mathrm{CP}$ que representa el 31\% de los alumnos con 210 de 368 con un nivel entre bueno y excelente. Para LAE 186 de 324 alumnos pertenecen al nivel bueno o excelente, LATIC con 66 de los 94 alumnos encuestados pertenecen al mismo rango y por último LAF y LAG cuentan con desempeños entre bueno y excelente de 170 de sus 291 encuestados y 64 de 123 alumnos respectivamente. También puede observarse que, en cuanto a género, solamente en el programa de LATIC es mayor el desempeño de los hombres, mientras que en todos los demás el desempeño de mujeres es más alto. 
RLCS, Revista Latina de Comunicación Social, 78, 309-328

[Investigación] DOI: 10.4185/RLCS-2020-1479| ISSN 1138-5820| Año 2020

Tabla 6. Correlaciones de Pearson entre género, dominio alumnos y maestros, estilo de aprendizaje, características de las TIC y nivel de satisfacción.

Correlaciones

\begin{tabular}{|c|c|c|c|c|c|c|c|}
\hline & & Género & $\begin{array}{l}\text { Dominio } \\
\text { de las TIC } \\
\text { que utiliza } \\
\text { la UACH }\end{array}$ & $\begin{array}{c}\text { Estilo de } \\
\text { Aprendizaje }\end{array}$ & $\begin{array}{c}\text { Características } \\
\text { de las TIC } \\
\text { usadas en } \\
\text { clases a } \\
\text { distancia por } \\
\text { COVID } \\
\end{array}$ & $\begin{array}{l}\text { Nivel de } \\
\text { satisfacción } \\
\text { del uso de } \\
\text { plataformas } \\
\text { virtuales } \\
\text { UACH } \\
\end{array}$ & $\begin{array}{c}\text { Evaluación } \\
\text { del dominio } \\
\text { del maestro } \\
\text { en clases en } \\
\text { línea }\end{array}$ \\
\hline Género & $\begin{array}{l}\text { Correlación de Pearson } \\
\text { Significancia (bilateral) } \\
\text { N }\end{array}$ & $\begin{array}{r}1 \\
1198\end{array}$ & $\begin{array}{r}-.008 \\
.770 \\
1198\end{array}$ & $\begin{array}{r}.026 \\
.366 \\
1198\end{array}$ & $\begin{array}{r}-.018 \\
.538 \\
1198 \\
\end{array}$ & $\begin{array}{r}-.081 \\
.005 \\
1198\end{array}$ & $\begin{array}{r}-.020 \\
.497 \\
1196\end{array}$ \\
\hline $\begin{array}{l}\text { Dominio de las TIC } \\
\text { que utiliza la UACH } \\
\text { Pearson }\end{array}$ & $\begin{array}{l}\text { Correlación de } \\
\text { Significancia (bilateral) } \\
\mathrm{N}\end{array}$ & $\begin{array}{r}-.008 \\
.770 \\
1198\end{array}$ & 1198 & $\begin{array}{r}-.097 \\
.001 \\
1198\end{array}$ & $\begin{array}{r}-.023 \\
.433 \\
1198\end{array}$ & $\begin{array}{r}-.577 \\
.000 \\
1198\end{array}$ & $\begin{array}{r}-.469 \\
.000 \\
1196 \\
\end{array}$ \\
\hline Estilo de aprendizaje & $\begin{array}{l}\text { Correlación de Pearson } \\
\text { Significancia (bilateral) } \\
\mathrm{N}\end{array}$ & $\begin{array}{r}-.026 \\
.366 \\
1198\end{array}$ & $\begin{array}{r}-.097 \\
.001 \\
1198\end{array}$ & $\begin{array}{r}1 \\
1198\end{array}$ & $\begin{array}{r}-.036 \\
.214 \\
1198\end{array}$ & $\begin{array}{r}-.064 \\
.026 \\
1198\end{array}$ & $\begin{aligned}-.038 \\
.191 \\
1196\end{aligned}$ \\
\hline $\begin{array}{l}\text { Características de las. } \\
\text { TIC usadas en las clases } \\
\text { a distancia por COVID }\end{array}$ & $\begin{array}{l}\text { Correlación de Pearson } \\
\text { Significancia (bilateral) } \\
\text { N }\end{array}$ & $\begin{array}{r}-.018 \\
.538 \\
1198\end{array}$ & $\begin{aligned}-.023 \\
.433 \\
1198\end{aligned}$ & $\begin{array}{r}-.036 \\
.214 \\
1198\end{array}$ & 1198 & $\begin{array}{r}-.024 \\
.397 \\
1198\end{array}$ & $\begin{array}{r}-.034 \\
.238 \\
1196\end{array}$ \\
\hline $\begin{array}{l}\text { Nivel de satisfacción del } \\
\text { uso de plataformas } \\
\text { virtuales UACH. }\end{array}$ & $\begin{array}{l}\text { Correlación de Pearson } \\
\text { Significancia (bilateral) } \\
\text { N }\end{array}$ & $\begin{array}{r}-.081 \\
.005 \\
1198 \\
\end{array}$ & $\begin{array}{r}-.577 \\
.000 \\
1198 \\
\end{array}$ & $\begin{array}{r}.064 \\
.026 \\
1198 \\
\end{array}$ & $\begin{array}{r}-.024 \\
.397 \\
1198 \\
\end{array}$ & $\begin{array}{r}1 \\
1198 \\
\end{array}$ & $\begin{array}{r}-.560 \\
.000 \\
1196 \\
\end{array}$ \\
\hline $\begin{array}{l}\text { Evaluación del dominio } \\
\text { del maestro en clases } \\
\text { en línea }\end{array}$ & $\begin{array}{l}\text { Correlación de Pearson } \\
\text { Significancia (bilateral) } \\
\mathrm{N}\end{array}$ & $\begin{array}{r}-.020 \\
.497 \\
1196 \\
\end{array}$ & $\begin{array}{r}-.469 \\
.000 \\
1196 \\
\end{array}$ & $\begin{array}{r}-.038 \\
.191 \\
1196 \\
\end{array}$ & $\begin{array}{r}-.034 \\
.238 \\
1196 \\
\end{array}$ & $\begin{array}{r}-.560 \\
.000 \\
1196 \\
\end{array}$ & 1196 \\
\hline
\end{tabular}

**La correlación es significativa en el nivel 0.01 (2colas)

* La correlación es significativa en el nivel 0.05 (2colas)

Fuente: Elaboración propia (2020)

Las correlaciones de Pearson positivas más significativas entre los indicadores de la tabla anterior muestran lo siguiente, 0.081 que muestra una relación baja entre el género y el nivel de satisfacción del uso de las plataformas virtuales en la FCA de la UACH, un 0.026 también entre el género y el estilo de aprendizaje. Con un 0.577 que demuestra una relación moderada entre el dominio de las TIC y el uso de las plataformas virtuales de la UACH, el 0.469 también moderada entre el dominio de las TIC y la evaluación del maestro en cursos en línea. Además, el 0.023 demostrando una relación baja entre el estilo de aprendizaje y las características de las TIC usadas en clases a distancia por COVID, así mismo el 0.560 de relación moderada entre el nivel de satisfacción del uso de las plataformas virtuales UACH y la evaluación del dominio del maestro en clases en línea. Mientras que la correlación negativa más relevante es entre el estilo de aprendizaje y el dominio de las TIC que utiliza la UACH con un -0.097 mostrando una relación inversa muy baja.

Tabla 7. Correlaciones de Pearson de los programas educativos con dominio, redes sociales, semestre, herramientas usadas, continuación de clases virtuales y lugar de conexión.

Correlaciones

\begin{tabular}{|c|c|c|c|c|c|c|c|c|}
\hline & & $\begin{array}{l}\text { Programa } \\
\text { educativo } \\
\text { al que } \\
\text { pertenece }\end{array}$ & $\begin{array}{l}\text { Dominio } \\
\text { de las } \\
\text { TIC que } \\
\text { utiliza la } \\
\text { UACH }\end{array}$ & $\begin{array}{c}\text { Redes } \\
\text { sociales } \\
\text { usadas } \\
\text { para clase } \\
\text { virtual } \\
\text { por } \\
\text { COVID }\end{array}$ & $\begin{array}{l}\text { Semestre } \\
\text { en curso }\end{array}$ & $\begin{array}{l}\text { Herramientas } \\
\text { usadas para } \\
\text { clase virtual } \\
\text { por COVID }\end{array}$ & $\begin{array}{l}\text { Continuaría } \\
\text { estudios } \\
\text { por clase } \\
\text { virtual } \\
\text { después del } \\
\text { COVID }\end{array}$ & $\begin{array}{l}\text { Lugar } \\
\text { donde se } \\
\text { conecta } \\
\text { para } \\
\text { clases } \\
\text { virtuales }\end{array}$ \\
\hline $\begin{array}{l}\text { Programa } \\
\text { educativo al } \\
\text { que pertenece }\end{array}$ & $\begin{array}{ll}\text { Correlación } & \text { de } \\
\text { Pearson } & \\
\text { Significancia } & \\
\text { (bilateral) } & \\
\text { N } & \end{array}$ & $\begin{array}{r}1 \\
1198\end{array}$ & $\begin{array}{r}.050 \\
.081 \\
1198\end{array}$ & $\begin{array}{r}-.076 * * \\
.008 \\
1198\end{array}$ & $\begin{array}{r}.015 \\
.614 \\
1198\end{array}$ & $\begin{array}{r}.053 \\
.068 \\
1198\end{array}$ & $\begin{array}{r}-.007 \\
.816 \\
1198\end{array}$ & $\begin{array}{r}-.039 \\
.182 \\
1198\end{array}$ \\
\hline
\end{tabular}


RLCS, Revista Latina de Comunicación Social, 78, 309-328

[Investigación] DOI: 10.4185/RLCS-2020-1479| ISSN 1138-5820| Año 2020

\begin{tabular}{|c|c|c|c|c|c|c|c|c|}
\hline $\begin{array}{lr}\text { Dominio } & \text { de } \\
\text { las TIC } & \text { que } \\
\text { utiliza } & \text { la } \\
\text { UACH } & \end{array}$ & $\begin{array}{l}\text { Correlación } \\
\text { Pearson } \\
\text { Significancia } \\
\text { (bilateral) } \\
\text { N }\end{array}$ & $\begin{array}{r}.050 \\
.081 \\
1198\end{array}$ & $\begin{array}{r}1 \\
1198\end{array}$ & $\begin{array}{r}-.016 \\
.586 \\
1198\end{array}$ & $\begin{array}{r}.103 * * \\
.000 \\
1198\end{array}$ & $\begin{array}{r}-.035 \\
.228 \\
1198\end{array}$ & $\begin{array}{r}.066^{*} \\
.023 \\
1198\end{array}$ & $\begin{array}{r}-.130 * * \\
.000 \\
1198\end{array}$ \\
\hline $\begin{array}{l}\text { Redes } \\
\text { sociales } \\
\text { usadas para } \\
\text { clase virtual } \\
\text { por COVID } \\
\end{array}$ & $\begin{array}{ll}\text { Correlación } & \text { de } \\
\text { Pearson } & \\
\text { Significancia } & \\
\text { (bilateral) } & \\
\mathrm{N} & \\
\end{array}$ & $\begin{array}{r}-.076 * * \\
.008 \\
1198\end{array}$ & $\begin{array}{r}-.016 \\
.586 \\
1198\end{array}$ & $\begin{array}{r}1 \\
1198\end{array}$ & $\begin{array}{r}.025 \\
.388 \\
1198\end{array}$ & $\begin{array}{r}-.041 \\
.153 \\
1198\end{array}$ & $\begin{array}{r}.031 \\
.282 \\
1198\end{array}$ & $\begin{array}{r}.006 \\
.839 \\
1198\end{array}$ \\
\hline $\begin{array}{l}\text { Semestre en } \\
\text { curso }\end{array}$ & $\begin{array}{l}\text { Correlación } \\
\text { Pearson } \\
\text { Significancia } \\
\text { (bilateral) } \\
\text { N }\end{array}$ & $\begin{array}{r}.015 \\
.614 \\
1198\end{array}$ & $\begin{array}{r}.103 * * \\
.000 \\
1198\end{array}$ & $\begin{array}{r}.025 \\
.388 \\
1198\end{array}$ & 1 & $\begin{array}{r}.052 \\
.071 \\
1198\end{array}$ & $\begin{array}{r}-.056 \\
.051 \\
1198\end{array}$ & $\begin{array}{r}-.008 \\
.772 \\
1198\end{array}$ \\
\hline $\begin{array}{l}\text { Herramientas } \\
\text { usadas para } \\
\text { clase virtual } \\
\text { por COVID }\end{array}$ & $\begin{array}{l}\text { Correlación } \\
\text { Pearson } \\
\text { Significancia } \\
\text { (bilateral) } \\
\text { N }\end{array}$ & $\begin{array}{r}.53 \\
.068 \\
1198\end{array}$ & $\begin{array}{r}-.035 \\
.228 \\
1198\end{array}$ & $\begin{array}{r}-.041 \\
.153 \\
1198\end{array}$ & $\begin{array}{r}.052 \\
.071 \\
1198\end{array}$ & $\begin{array}{r}1 \\
1198\end{array}$ & $\begin{array}{r}-.013 \\
.642 \\
1198\end{array}$ & $\begin{array}{r}-.020 \\
.488 \\
1198\end{array}$ \\
\hline $\begin{array}{l}\text { Continuaría } \\
\text { estudios por } \\
\text { clase virtual } \\
\text { después del } \\
\text { COVID }\end{array}$ & $\begin{array}{l}\text { Correlación de } \\
\text { Pearson } \\
\text { Significancia } \\
\text { (bilateral) } \\
\text { N }\end{array}$ & $\begin{array}{r}-.007 \\
.816 \\
1198\end{array}$ & $\begin{array}{r}.066^{*} \\
.023 \\
1198\end{array}$ & $\begin{array}{r}.031 \\
.282 \\
1198\end{array}$ & $\begin{array}{r}-.056 \\
.051 \\
1198\end{array}$ & $\begin{array}{r}-.013 \\
.642 \\
1198\end{array}$ & $\begin{array}{r}1 \\
1198\end{array}$ & $\begin{array}{r}-.075 * * \\
.010 \\
1198\end{array}$ \\
\hline $\begin{array}{l}\text { Lugar donde } \\
\text { se conecta } \\
\text { para clases } \\
\text { virtuales }\end{array}$ & $\begin{array}{l}\text { Correlación } \\
\text { Pearson } \\
\text { Significancia } \\
\text { (bilateral) } \\
\text { N }\end{array}$ & $\begin{array}{r}-.039 \\
.182 \\
1198\end{array}$ & $\begin{array}{r}-.130 * \\
.000 \\
1198\end{array}$ & $\begin{array}{r}.006 \\
.839 \\
1198\end{array}$ & $\begin{array}{r}-.008 \\
.772 \\
1198\end{array}$ & $\begin{array}{r}-.020 \\
.488 \\
1198\end{array}$ & $\begin{array}{r}-.075 * \\
.010 \\
1198\end{array}$ & 1198 \\
\hline
\end{tabular}

* La correlación es significativa en el nivel 0.05 (2colas)

Fuente: Elaboración propia (2020)

La tabla de correlaciones para los 7 indicadores mencionados, muestra que existe una correlación significativa positiva de 0.05 , relación muy baja entre el programa educativo al que pertenece el alumno y las herramientas utilizadas, así como el dominio de las TIC que usa la institución educativa. En cuanto al dominio de las TIC tiene una correlación significativa positiva de 0.1 también una relación muy baja con el semestre en el cual está inscrito actualmente, por lo que respecta a la continuación de clases virtuales aún sin la contingencia COVID-19 existe una correlación significativa positiva de 0.03 con relación muy baja con el uso de las redes sociales utilizadas para clases por la contingencia. También existe una correlación significativa positiva del 0.6 de relación moderada entre si continuaría utilizando clases en línea y el dominio que tiene en el desempeño de las TIC. Por último, existe una correlación significativa negativa de -0.13 entre el dominio de las TIC y el lugar donde tiene su conexión a internet, mostrando una relación inversa muy baja.

Tabla 8. Ventajas que ofrecen las TIC

\begin{tabular}{|l|c|c|c|c|c|}
\hline & C.P. & L.A.E. & L.A.T.I.C. & L.A.F. & L.A.G. \\
\hline Disponibilidad de información & 265 & 313 & 87 & 246 & 105 \\
\hline Mayor interacción con el profesor & 61 & 72 & 39 & 57 & 35 \\
\hline Mayor interacción con los compañeros & 51 & 65 & 18 & 41 & 16 \\
\hline Mayor aprendizaje de la materia & 32 & 42 & 9 & 18 & 11 \\
\hline Aprendizaje colaborativo & 100 & 133 & 37 & 78 & 46 \\
\hline Mayor comunicación (profesor/alumnos) & 83 & 80 & 25 & 72 & 31 \\
\hline
\end{tabular}

Fuente: Elaboración propia (2020) 
De la tabla anterior se aprecia que el $85 \%$ de los alumnos consideran como ventaja de las clases en línea la disponibilidad de la información, un $22 \%$ cree que hay una mayor interacción con el profesor y $16 \%$ con los compañeros. Sólo el $9 \%$ piensa que tiene mayor aprendizaje de la materia, mientras un $33 \%$ considera una ventaja en el aprendizaje colaborativo. Por último, un $24 \%$ de los encuestados opina que existe una mayor comunicación entre el profesor y los alumnos.

Tabla 9. Desventajas que ofrecen las TIC

\begin{tabular}{|l|c|c|c|c|c|}
\hline & C.P. & L.A.E. & L.A.T.I.C. & L.A.F. & L.A.G. \\
\hline Fallas en la conexión & 288 & 301 & 82 & 231 & 107 \\
\hline Menor comunicación (profesor/alumnos) & 169 & 205 & 35 & 146 & 52 \\
\hline Acceso a muchas distracciones & 182 & 211 & 48 & 165 & 71 \\
\hline Menor aprendizaje & 187 & 209 & 30 & 172 & 62 \\
\hline Falta de interacción con los compañeros & 147 & 136 & 30 & 122 & 42 \\
\hline Dificultad para manejarlas & 97 & 98 & 25 & 63 & 36 \\
\hline $\begin{array}{l}\text { Falta de herramientas tecnológicas (Computadora } \\
\text { o internet) }\end{array}$ & 88 & 82 & 38 & 57 & 38 \\
\hline
\end{tabular}

Fuente: Elaboración propia (2020)

En cuanto a las desventajas, la tabla muestra los siguientes resultados: el $84 \%$ de los encuestados tuvo problemas de conexión, el $51 \%$ de los alumnos sintió menos comunicación con el profesor y el $57 \%$ considera que tiene acceso a muchas distracciones. Mientras un 55\% cree que su aprendizaje es menor y el $40 \%$ que falta interacción con sus compañeros de clase, también el 27\% comenta que tiene dificultad para manejarlas y el $25 \%$ no cuenta con las herramientas tecnológicas para la clase en línea como lo es el equipo de cómputo y la conexión a internet.

\section{Discusión}

Los resultados de la investigación manifiestan que los universitarios de la institución ostentan una correlación positiva hacia las tecnologías de información y comunicación, que se muestra en varios aspectos estudiados: estilo de aprendizaje, dominio en el uso de las TIC, herramientas, programa educativo, nivel de satisfacción de contar con los recursos tecnológicos apropiados, entre otros. Uno de los hallazgos más importantes de esta investigación fue que los estudiantes tienen muy buen dominio de las TIC para manejar las herramientas tecnológicas utilizadas durante la contingencia sanitaria, así mismo una correlación positiva entre el dominio de las TIC y el uso de las plataformas virtuales de la UACH, entre el estilo de aprendizaje y las características de las TIC usadas en clases a distancia.

Mominó y Sigáles (2016) mencionan que en el contexto educativo es donde corresponde hacerse más evidente el conjunto de transformaciones tecnológico-sociales porque en este contexto es donde se producen y socializan los individuos. En el mundo de hoy y ante la situación actual de la pandemia del COVID-19, el poder acceder a las tecnologías de la información y comunicaciones (TIC) fue una necesidad significativa para participar en un contexto cada vez más dependiente de la tecnología. Las TIC son un elemento dinamizador fundamental en la sociedad y organizaciones de cualquier tipo. La Universidad Autónoma de Chihuahua en México pasó de la educación tradicional al modelo de educación a distancia a través de herramientas tecnológicas educativas. Hernández et ál. (2020) señalan que, ante este contexto, los maestros y estudiantes deberán adquirir diferentes competencias y tomar roles diferentes a los que tradicionalmente han realizado. Las tecnologías de la información y comunicación en la educación posibilitan varias alternativas y a la vez representan un enorme conjunto de desafíos, a través de ellas se puede producir conocimiento amplio y flexible, relacionándose con aplicación, innovación, negociación, encaminar, adoptar, difundir, establecer, 
incrementar, en fin; una serie de conceptos que se transpongan a la no linealidad del aprendizaje que se produce a partir de la utilización de estas, dando arranque al estudio de aprendizajes, a la corrección, deconstrucción, etcétera. (Cobo, 2016)

Las TIC impulsan el desarrollo de los procesos de enseñanza y aprendizaje colocando a disposición de los estudiantes diferentes alternativas para conseguir de manera sencilla los objetivos de aprendizaje (Aguaded y Cabero, 2014). Motivo por el cual, son consideradas en esta investigación como herramientas que brindan muchas opciones de uso tanto para el estudiante como al docente en el momento de impartir conocimientos y de esta manera cumplir los objetivos del quehacer educativo.

Las TIC al igual que las demás actividades humanas se convierten en una herramienta cada vez más necesaria en las instituciones educativas donde pueden realizar variadas funcionalidades, para lograr el trabajo colaborativo y lo más importante en la educación presente, conseguir que el aprendizaje sea significativo. (Queiruga, Sáiz y Montero, 2018)

En diversos estudios parece ser una constante el que existe conciencia de las ventajas de las TIC, pero su uso no termina por consolidarse. Según Pelgrum (2001), aún en ambientes favorables, la mayoría de las personas dedicadas al sector de la educación consideran la falta de equipo de cómputo como uno de los mayores problemas. Ottenbreit-Leftwich et ál. (2010) señalan que en algunas ocasiones se cuenta que profesores con opiniones positivas hacia la tecnología las adopten en su práctica profesional. En esta investigación la mayor ventaja fue contar con la información disponible en todo momento y como segunda opción señalada fue el aprendizaje colaborativo y la desventaja más mencionada fueron las fallas en la conexión a internet.

Campos y Ramírez (2018) en un estudio señalan que la edad, la actitud, la familiaridad y los conocimientos relacionados con las TIC no son obstáculos a vencer para integrarlas en los programas educativos; a los participantes parecía afectarles de modo significativo el uso y la forma de utilizar la tecnología. En este estudio coincide en que la edad y el perfil académico no eran los problemas, las barreras principales fueron la falta de equipo, poca capacitación, resistencia a adoptar nuevas tecnologías o métodos entre otras, situación derivada de la crisis por la pandemia del COVID-19. García (2014) señala los usuarios utilizan en mayor o menor medida las herramientas digitales, y se insertan más intensamente en su manejo, no en función de la edad, sino del contexto, motivaciones e intereses, en los que el factor actitud influye más que la experiencia. Los estudiantes y profesores se encuentran ante la problemática de adaptarse a estos cambios y tener que aprender nuevas formas de transmitir el conocimiento. Esta etapa de transición tendrá un cambio en las instituciones educativas debido a que el proceso de aprendizaje será diferente al tradicional porque el uso de las TIC en el entorno educativo a través de la educación a distancia y la adecuada utilización didáctica supone un reto sin precedentes.

\section{Conclusiones}

Debido a las medidas de confinamiento y distanciamiento social, ante la contingencia COVID-19, las tecnologías han cobrado aún más relevancia, y para algunos, tanto maestros como alumnos, han tenido que involucrarse a marchas forzadas para la elaboración de materiales, uso de portales Web y plataformas tecnológicas utilizadas en el proceso de enseñanza aprendizaje.

Con base en los resultados, se puede confirmar que las herramientas que los estudiantes están utilizando más en las clases a distancia son la plataforma Moodle donde el maestro les comparte materiales de los cursos, clases en videoconferencia a través de Google Meet y para la elaboración de 
trabajos consultan información por medio de los diferentes buscadores. Los estudiantes caracterizan las TIC como accesibles y flexibles.

Existe una correlación directa entre el dominio de las TIC y el uso de las plataformas virtuales utilizadas en la UACH y entre el nivel de satisfacción del uso de las plataformas virtuales UACH y la evaluación del dominio del maestro en clases en línea. Con esto se confirma que las TIC utilizadas en la UACH son del dominio de los estudiantes.

Sin embargo, al analizar la relación presente entre si continuarían tomando clases en línea y el dominio que tiene en el desempeño de las TIC, se encontró una relación significativa positiva moderada, esto indica que entre más dominio tienen los alumnos en su desempeño con las TIC, más les interesa seguir tomando clases en línea.

Los teléfonos inteligentes, el correo electrónico y la red social de WhatsApp fueron señaladas como parte de las herramientas tecnológicas más utilizadas por parte de los alumnos, las cuales facilitaron esta transición de la educación tradicional a la educación a distancia, gracias a estas fue posible no perder la comunicación entre profesores y alumnos y poder llegar a aquellos estudiantes sin computadora y sin acceso a internet; no solo por provenir de lugares en donde no existe este servicio sino por la situación económica familiar ante la pandemia.

La disponibilidad de información y aprendizaje colaborativo son las ventajas que los estudiantes consideran de las TIC como herramientas para llevar a cabo la interacción entre los diversos participantes en el proceso de enseñanza aprendizaje. De las desventajas que se identificaron fue el de fallas en la conexión de Internet y el que tienen acceso a muchas distracciones durante la clase y consideran que tienen un menor aprendizaje.

\section{Recomendaciones}

Teniendo en cuenta que esta pandemia es una coyuntura para identificar las habilidades que todo estudiante y docente requieren en el proceso de enseñanza aprendizaje, precisamente en crisis como esta, donde fue necesario adentrarse en la educación a distancia a través del uso de las tecnologías, se recomienda continuar con la capacitación a docentes de las distintas disciplinas donde apliquen de manera eficiente el uso de las mismas en el quehacer docente, donde eleven la productividad en el uso de Internet y las herramientas necesarias en la educación a distancia. Para que, de esta manera, los docentes adquieran las competencias necesarias para lograr una comunicación efectiva con los estudiantes por medio de las tecnologías.

\section{Bibliografía}

Aguaded, J. I., y Cabero, J. (2014). Avances y retos en la promoción de la innovación didáctica con las tecnologías emergentes e interactivas. Educar, 8(50), 67-83.

http://dx.doi.org/10.5565/rev/educar.691

Astudillo, M., Pinto, B., Arboleda, J., y Anchundia, Z. (2018) Aplicación de las Tic como herramienta de aprendizaje en la Educación Superior. Revista Científica Mundo de la $\begin{array}{lllll}\text { Investigación } & \mathrm{y} & \text { el } & \text { Conocimiento. } & 2(2) .\end{array}$ https://doi.org/10.26820/recimundo/2.(2).2018.585-598

Avello-Martínez, R. y Duart, J.M. (2016). Nuevas tendencias de aprendizaje colaborativo en elearning: Claves para su implementación efectiva. Estudios pedagógicos (Valdivia), 42(1), 271282. 
Cacheiro, M. (2014). Educación y Tecnología: Estrategias didácticas para la integración de las TIC. Madrid: Editorial UNED.

Cacheiro, G.M.L. (2018). Educación y tecnología: estrategias didácticas para la integración de las TIC Ciencias Sociales y Jurídicas. Editorial UNED.

Campos, H., y Ramírez, M. (2018). Las TIC en los procesos educativos de un centro público de investigación. Apertura, 10(1), 56-70. http://dx.doi.org/10.18381/Ap.v10n1.1160

Cobo, C. (2016). La Innovación Pendiente. Reflexiones (y provocaciones) sobre educación, tecnología y conocimiento. Montevideo: Colección Fundación Ceibal.

Consejo de Salubridad General. (2020). Requisito Certificación Hospitales.pdf. Diario Oficial de la Federación.

http://www.csg.gob.mx/descargas/pdf/index/informacion_relevante/Requisito_Certificacion_Hos pitales.pdf

Coyago, A. P. R., Puente, E. y Jiménez, G. A. M. (2017). Uso de las tecnologías de la información en la educación superior. INNOVAResearch Journal, 2(1), 99-112. https://doi.org/10.33890/innova.v2.n1.2017.154

Fernández-Cruz, F. J. y Fernández-Díaz, M. J. (2016) Los docentes de la Generación Z y sus competencias digitales. Comunicar, 46(24), 103. https://doi.org/10.3916/C46-2016-10

Gallego Torres, R. (2017). Study telling, base teórica de una nueva comunicación para el e-learning. In Caleidoscopio Educativo (pp. 135-144). Madrid: Global Knowledge Academics.

García, A.L. (2014). Bases, mediaciones y futuro de la educación a distancia en la sociedad digital. Madrid: Síntesis.

Gobierno de México. (2020). Semáforo. Coronavirus. https://coronavirus.gob.mx/semaforo/

Hernández, J. J, Jiménez, Y. y Rodríguez, E. (2020). Más allá de los procesos de enseñanzaaprendizaje tradicionales: construcción de un recurso didáctico digital. Revista Iberoamericana para la Investigación y Desarrollo Educativo. Vol. 10 (20). Recuperado de: https://doi.org/10.23913/ride.v10i20.622

Herrero, C., \& Brown, M. (2010). Distributed Cognition. Revista de Psicodidáctica, 15(2), 253-268. https://ojs.ehu.eus/index.php/psicodidactica/article/view/816

Mominó, J. M., y Sigáles, C. (2016). El impacto de las TIC en la educación. Más allá de las promesas. Barcelona: UOC Ediciones.

Moreno, R. H. (2016). Incorporación de las TIC en las prácticas educativas: el caso de las herramientas, recursos, servicios y aplicaciones digitales de Internet para la mejora de los procesos de aprendizaje escolar. Reencuentro. Análisis de Problemas Universitarios, (72),71-92. [fecha de Consulta 1 de Junio de 2020]. ISSN: 0188-168X. https://www.redalyc.org/articulo.oa?id=340/34051292006 
RLCS, Revista Latina de Comunicación Social, 78, 309-328

[Investigación] DOI: 10.4185/RLCS-2020-1479| ISSN 1138-5820| Año 2020

Ottenbreit-Leftwich, A., Glazewski, K., Newby, T. y Ertmer, P. (2010). Teacher value beliefs associated with using technology: Addressing professional and student needs. Computers \& Education, vol. 55, 1321-1335. http://dx.doi.org/10.1016/j.compedu.2010.06.002

Queiruga, M., Sáiz, M., y Montero, E. (2018). Transformar el aula en un escenario de aprendizaje significativo. Revista Educativa Hekademos, 24(11). 7-18. Recuperado de https://dialnet.unirioja.es/servlet/articulo?codigo $=6542599$

Pelgrum, W. (2001). Obstacles to the integration of ICT in education: Results from a worldwide educational assessment. Computers \& Education, vol. 37, 163-178. Recuperado de http://users.ntua.gr/vvesk/ictedu/article5_pelgrum.pdf

Perkins, M. y Pfaffman, J. (2006). Using a course management system to improve classroom communication. Science Teacher, 73(7), 33-37.

Secretaría de Educación Pública. (2020). Acuerdo número 02/03/20 [Diario Oficial de la Federación]. $\quad$ SEGOB. Recuperado de https://www.dof.gob.mx/nota_detalle.php?codigo=5589479\&fecha=16/03/2020

Severin, E. (2010). Tecnologías de la Información y la Comunicación (TIC) en Educación. Banco Interamericano de Desarrollo. España.

Silva, M., García, T., Guzmán, T. y Chaparro, R. (2016). Estudio de herramientas Moodle para desarrollar habilidades del siglo XXI. Campus Virtuales, 5(2), 58-69. http://uajournals.com/ojs/index.php/campusvirtuales/article/view/126

Sierra, I. y Carrascal, N. (2011). Concepciones y estrategias docentes en entornos virtuales, fundamentos para una tipología. Monteria: Universidad de Córdoba.

Smith, Sh. y Xu, D. (2016). How do online course design features influence student performance? Computers \& Education, 95, 270-284. http://dx.doi.org/10.1016/j.compedu.2016.01.014.

St-Pierre, A. (2001). Pedagogía e Internet: aprovechamiento de las nuevas tecnologías. México: Trillas.

Vázquez, M. H. y Burrial, A. T. (2017). Factores que influyen en el aprendizaje mixto (blendedlearning) y colaborativo en Moodle en Didáctica de las Ciencias Experimentales en el Grado de Maestro en Educación Primaria. In Propuesta de Innovación Educativa en la Sociedad de la Información (48-60). Adaya Press.

Vuopala, E., Hyvönen, P. y Järvelä, S. (2016). Interaction forms in successful collaborative learning in virtual learning environments. ActiveLearning in Higher Education, 17(1), 25-38. https://doi.org/10.1177/1469787415616730

Yanacón-Atía, R. y Menini. M. (2018). Indicadores colaborativos individuales y grupales para Moodle. $\quad$ Campus $\quad$ Virtuales, $\quad 7(1), \quad 125-139$. http://uajournals.com/ojs/index.php/campusvirtuales/article/view/309 


\title{
AUTORES/AS
}

\begin{abstract}
Alma Lilia Sapién Aguilar
Doctora en Administración con Postdoctorado en Ciencias Humanas. Profesora Investigadora de la Facultad de Contaduría y Administración de la Universidad Autónoma de Chihuahua. Miembro del Sistema Nacional de Investigadores Nivel I de CONACYT. Las líneas de investigación: innovación tecnológica, comportamiento organizacional, administración con valores y educación.
\end{abstract}

lsapien@uach.mx

Orcid ID: http://orcid.org/0000-0001-7222-2612

\section{Laura Cristina Piñón Howlet}

Doctora en Administración con Postdoctorado en Ciencias Humanas. Profesora Investigadora de la Facultad de Contaduría y Administración de la Universidad Autónoma de Chihuahua. Miembro del Sistema Nacional de Investigadores Nivel I de CONACYT. Las líneas de investigación: innovación tecnológica, comportamiento organizacional, administración con valores y educación.

lpinon@uach.mx

Orcid ID: http://orcid.org/0000-0002-1176-2567

\section{María Del Carmen Gutiérrez Diez}

Doctora en Administración con Postdoctorado en Ciencias Humanas. Profesora Investigadora de la Facultad de Contaduría y Administración de la Universidad Autónoma de Chihuahua. Miembro del Sistema Nacional de Investigadores Nivel I de CONACYT. Las líneas de investigación: innovación tecnológica, comportamiento organizacional, administración con valores y educación.

cgutierr@uach.mx

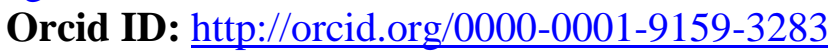

\section{José Luis Bordas Beltrán}

Doctor en Administración; labora en la Universidad Autónoma de Chihuahua en la Facultad de Contaduría y Administración en México. Miembro del Sistema Nacional de Investigadores Nivel I de CONACYT. Las líneas de investigación: Comunicación, Cultura y Gestión de Tecnología.

jbordas@uach.mx

Orcid ID: https://orcid.org/0000-0003-1465-3662 


\section{Anexo.}

\section{Cuestionario}

1. Sexo: $\quad \square$ Mujer $\quad \square$ Hombre

2. Edad: $\square$ Entre 17 y 19 años

$\square$ Entre 20 y 22 años

$\square$ Entre 23 y 25 años

Más de 25 años

3. Estado civil: $\square$ Soltero $\square$ Casado

4. Seleccione su Programa Educativo:

$\square$ Licenciado en Administración de Empresas

$\square$ Contador Público

$\square$ Licenciado en Administración Financiera

$\square$ Licenciado en Administración Gubernamental

$\square$ Licenciado en Administración de Tecnologías de Información y Comunicación

5. Semestre (en caso de llevar materias de diferentes semestres, favor de indicar el semestre al que pertenezca la materia de menor avance):

6. ¿Cuánto considera que conoce sobre el COVID-19?
$\square$ Nada
$\square$ Regular $\square$ Mucho

7. En su casa o lugar de trabajo cuenta con conexión a Internet
$\square \mathrm{Si}$
$\square$ No

8. ¿Cuál es su estilo de aprendizaje predominante?
$\square$ Auditivo
$\square$ Visual
$\square$ Kinestésico (Por tacto)
Desconozco

9. ¿Cómo considera que es su dominio en el uso de las TIC con las herramientas que está utilizando la Universidad ante la contingencia del COVID-19?
$\square$ Excelente
$\square$ Muy bueno
$\square$ Bueno
Regular $\square$ Malo

10. ¿Qué tan difícil ha sido para ti manejar las herramientas tecnológicas para las clases virtuales?
$\square$ Muy difícil
$\square$ Difícil
$\square$ Neutral
$\square$ Fácil
$\square$ Muy fácil

11. ¿Los profesores que imparten las clases virtuales dominan las herramientas tecnológicas utilizadas en su clase?
$\square$ Nada
Regular
Mucho

12. ¿Cuáles herramientas tecnológicas están utilizando en las clases ante la contingencia del COVID-19?

$\square$ Plataforma Moodle

Video conferencias por medio de la plataforma Meet

Video conferencias por medio de la plataforma Zoom

WhatsApp

$\square$ Facebook

$\square$ Nube computacional (Google Drive, box, One Drive, Dropbox)

$\square$ Otra 
RLCS, Revista Latina de Comunicación Social, 78, 309-328

[Investigación] DOI: 10.4185/RLCS-2020-1479| ISSN 1138-5820| Año 2020

13. ¿Qué nivel de satisfacción tiene sobre el uso de plataformas virtuales utilizadas en la Universidad?

$\square$ Completamente satisfecho

$\square$ Bastante satisfecho

$\square$ Indiferente

$\square$ Bastante insatisfecho

Completamente insatisfecho

14. ¿Con que frecuencia hace uso de las plataformas virtuales en sus clases?

$\square$ Una vez al día

$\square$ Varias veces al día

$\square$ Una vez por semana

$\square$ Todos los días

$\square$ Los fines de semana

15. ¿Cuál es el motivo por el cual utiliza las plataformas virtuales?
$\square$ Revisar material
$\square$ Realizar una actividad
$\square$ Participar en un foro
$\square$ Chat
$\square$ Subir una tarea
$\square$ Ver videos
$\square$ Otro

16. ¿Qué característica describen mejor a las tecnologías de información y comunicaciones utilizadas en sus clases virtuales? (Seleccione todas las que correspondan)
$\square$ Dinámicas
$\square$ Accesibles
Sencillas
Flexibles
Complicadas

17. ¿Con que frecuencia hace uso de videoconferencias en sus clases con sus profesores?
$\square$ Una vez al día
$\square$ Varias veces al día
$\square$ Una vez por semana
$\square$ Todos los días
$\square$ Los fines de semana

18. ¿Consideras que el maestro establece ambientes propicios para el aprendizaje utilizando las Tecnologías de Información y comunicaciones (TIC)?

$\square$ Nunca $\square$ Casi nunca $\square$ Ocasionalmente $\square$ Casi todos los días $\square$ Todos los días

\begin{tabular}{|c|c|c|c|c|c|}
\hline & $\begin{array}{l}\text { Totalmente } \\
\text { de acuerdo }\end{array}$ & $\begin{array}{l}\text { De } \\
\text { acuerdo }\end{array}$ & $\begin{array}{c}\text { Ni de acuerdo } \\
\text { ni en } \\
\text { desacuerdo }\end{array}$ & Desacuerdo & $\begin{array}{l}\text { Totalmente en } \\
\text { desacuerdo }\end{array}$ \\
\hline $\begin{array}{l}\text { 19. Utilizar las TIC facilita el } \\
\text { aprendizaje de la clase. }\end{array}$ & & & & & \\
\hline $\begin{array}{l}\text { 20. Utilizar las TIC facilita realizar un } \\
\text { trabajo o tarea. }\end{array}$ & & & & & \\
\hline $\begin{array}{l}\text { 21. Es importante el uso de TIC dentro } \\
\text { de las materias que se imparten en la } \\
\text { Facultad de Contaduría y } \\
\text { Administración }\end{array}$ & & & & & \\
\hline $\begin{array}{l}\text { 22. ¿Considera que el uso de las TIC } \\
\text { propicia una mejor comunicación } \\
\text { entre profesor y alumno? }\end{array}$ & & & & & \\
\hline $\begin{array}{l}\text { 23. ¿Considera que las clases mejoraran } \\
\text { con el uso de las TIC? }\end{array}$ & & & & & \\
\hline
\end{tabular}


RLCS, Revista Latina de Comunicación Social, 78, 309-328

[Investigación] DOI: 10.4185/RLCS-2020-1479| ISSN 1138-5820| Año 2020

\begin{tabular}{|c|c|c|c|c|c|}
\hline & $\begin{array}{c}\text { Nada } \\
\text { frecuente }\end{array}$ & $\begin{array}{c}\text { Poco } \\
\text { frecuente }\end{array}$ & Frecuentemente & $\begin{array}{l}\text { Bastante } \\
\text { frecuente }\end{array}$ & $\begin{array}{c}\text { Muy } \\
\text { frecuente }\end{array}$ \\
\hline $\begin{array}{l}\text { 24. ¿Has establecido comunicación online con } \\
\text { compañeros de clase para realizar alguna } \\
\text { actividad académica? }\end{array}$ & & & & & \\
\hline $\begin{array}{l}\text { 25. ¿Cuántas veces el profesor te encarga } \\
\text { trabajos o tareas en donde tengas que } \\
\text { hacer uso de la TIC? }\end{array}$ & & & & & \\
\hline $\begin{array}{l}\text { 26. ¿Utilizas la computadora y/o otras } \\
\text { tecnologías de la información cuando } \\
\text { realizas presentaciones en clase? }\end{array}$ & & & & & \\
\hline
\end{tabular}

27. ¿Seleccione los recursos que más utiliza para conectarse a las clases virtuales?
$\square$ Computadora de escritorio - PC
$\square$ Laptop / notebook
$\square$ Tablet
$\square$ Celular (Android, Apple, BlackBerry)
Otros

28. Seleccione la red social que más utiliza para uso de sus clases ante la contingencia del COVID-19
$\square$ Facebook
$\square$ WhatsApp
$\square$ Instagram
$\square$ YouTube
$\square$ Twitter
$\square$ No utilizo redes sociales

29. ¿Cuáles ventajas ofrecen las Tecnologías de Información y Comunicaciones (TIC) en sus clases virtuales ante la contingencia sanitaria COVID-19? (Seleccione todas las que correspondan)

$\square$ Disponibilidad de información

$\square$ Mayor interacción con el profesor

$\square$ Mayor interacción con los compañeros

$\square$ Mayor aprendizaje de la disciplina

$\square$ Aprendizaje colaborativo

$\square$ Mayor comunicación (profesor/alumnos)

30. ¿Cuáles desventajas ofrecen las Tecnologías de Información y Comunicaciones (TIC) en sus clases virtuales ante la contingencia sanitaria COVID-19? (Seleccione todas las que correspondan)

$\square$ Fallas en la conexión

$\square$ Menor comunicación (profesor/alumnos)

Acceso a muchas distracciones

$\square$ Menor aprendizaje

$\square$ Falta de interacción con los compañeros

$\square$ Dificultad para manejarlas 